\title{
Löw, Martina; Sayman, Volkan; Schwerer, Jona; Wolf, Hannah (Hrsg.) (2021): Am Ende der Globalisierung. Über die Refiguration von Räumen
}

\author{
Markus Hesse \\ Eingegangen: 13. Juli 2021 - Angenommen: 3. August 2021 - Online veröffentlicht: 30. August 2021
}

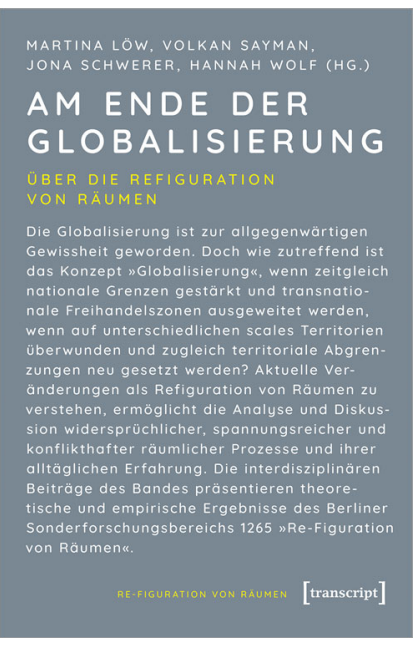

Globalisierung als zeitweise hegemoniales Konzept der sozialwissenschaftlichen Raum- und Gesellschaftsanalyse der 1990er- und 2000er-Jahre wird seit geraumer Zeit kritisch diskutiert und hinterfragt. Dies gilt beispielsweise bezüglich ihres vermeintlich alternativlosen Stellenwerts als Zeitdiagnose und Zukunftsentwurf (vgl. Sheppard 2016) oder hinsichtlich ihrer politischen Implikationen und Widersprü-

Prof. Dr. Markus Hesse, Department für Geographie und Raumplanung, Universität Luxemburg, 11 Porte des Sciences, 4366 Eschsur-Alzette, Luxemburg

markus.hesse@uni.lu

(c) (1) ( $) 2021$ Hesse; licensee oekom verlag. This Open Access article is published under a Creative Commons Attribution 4.0 International License. che, mit denen sie zwangsläufig in Konflikt zur demokratischen Verfasstheit liberaler Gesellschaften gerät (vgl. Rodrik 2011). Das vorliegende Buch reiht sich ein in diese jüngere Diskurslinie. Die Herausgeber/-innen haben sich zum Ziel gesetzt, einen eigenen Akzent im Globalisierungskontext zu setzen, unter anderem inspiriert durch die in der Corona-Krise sichtbar gewordene Vulnerabilität und Disruption globaler und internationaler Vernetzungen. Dabei wird nicht in Frage gestellt, dass Globalisierungsdynamiken künftig weiter Bestand haben. Im Sinne eines besseren Verständnisses dieser Prozesse und ihrer räumlichen Implikationen soll jedoch erstens aus den ,ewigen Schleifen“ der Gegenüberstellung von ,global und lokal“ (S. 10) ausgebrochen werden. Stattdessen werden Globalisierung einerseits und territoriale Schließung andererseits als ,zueinander in Spannung stehende Phänomene“ (S. 10) in sich wandelnden spätmodernen Gesellschaften verstanden. Zweitens wird dieser Wandel grundlegend als räumlicher Wandel aufgefasst.

Das Buch ist im Rahmen eines durch die Deutsche Forschungsgemeinschaft (DFG) geförderten Sonderforschungsbereichs entstanden; verfolgt wird das Konzept der „Refiguration von Räumen“ zur Beschreibung spätmoderner Gesellschaften. Das Konzept basiert auf der Absicht, das nach Auffassung der Autorinnen und Autoren zu eng gefasste analytische Konzept von einer Globalisierung zu pluralisieren und stattdessen von vielfältigen Mustern und Formen globaler Vernetzungen und daraus resultierenden Abhängigkeitsverhältnissen auszugehen. Refiguration wird dabei als Konzept verstanden, „mit dem empirisch nach den Varianten und vor allem den Verflechtungen im sozialen Wandel gefragt wird (wobei gleichzeitig doch Gemeinsamkeiten wie z.B. die digitalisierte Mediatisierung vorausgesetzt werden)“ (S. 11). Dies lenkt den Blick zum Beispiel auf 
die Dualität von global cities einerseits und abgehängten Regionen andererseits. Auch sollen die vielschichtigen, bei Weitem nicht nur ökonomischen Verflechtungen im Globalisierungskontext thematisiert werden.

Das Buch enthält 18 Beiträge von Autorinnen und Autoren aus einem weiten disziplinären Feld von Soziologie, Geographie und Architektur/Stadtplanung, die in fünf $\mathrm{Ab}$ schnitten thematisch sortiert sind. Auf eine Einleitung der Herausgeberinnen und Herausgeber folgt im ersten $\mathrm{Ab}$ schnitt (,Von der Globalisierung zur Refiguration“) die Darlegung der konzeptionellen Grundlage mit Bezug auf die Refiguration von Räumen. Diese Abhandlung wird unterstützt durch ein Virtual Essay mit Fotos aus verschiedenen Ländern, die den Prozess der „Verheimatung“ illustrieren; dieser Begriff meint die Verortung von Heimat jenseits der eigenen geographischen Herkunft. Im zweiten Abschnitt folgen drei Aufsätze zur „Verräumlichung von Politiken", in denen es um Nichtregierungsorganisationen und Interessengruppen in der Wohn- und Asylpolitik geht sowie um die „Infrastrukturierung von Wissensräumen“. In Wissensräumen werden ,,je spezielle Modelle, Theorien, Methoden, Techniken und Werkzeuge hergestellt und eingesetzt, mit denen Politik gemacht wird" (S. 109). Schließlich geht es um die Erkundung von Grenzinfrastrukturen und Politiken der Grenze im globalen Kontext. Der nachfolgende Abschnitt handelt von der „Refiguration digitalisierter Räume“ und enthält fünf Beiträge, die sich mit dem Aufkommen digitaler Instrumente zum Entwurf, zur Gestaltung oder Kontrolle von räumlichen Prozessen und Artefakten befassen, etwa Kontrollzentralen, smarte Apartmentkomplexe oder digitales Entwerfen und Visualisieren in der Stadtplanung. In einem Abschnitt unter dem Titel „Globallokales Raumwissen“ werden empirische und konzeptionelle Arbeiten zur globalen Mittelklasse, zu Imaginationen der Globalisierung (mit einer dezidierten Infragestellung des Buchtitels), globalen Produktionsnetzwerken oder zur Rolle von Öffentlichkeiten in Rotterdam und Berlin präsentiert. Ein weiterer Beitrag handelt vom „Homemaking“ in Berliner Unterkünften für Geflüchtete.

Ist das Spektrum an Themen und Zugängen der einzelnen Beiträge zu ihrem jeweiligen Gegenstand doch recht groß, binden die beiden Schlussbeiträge unter dem Rubrum „Rückblick und Ausblick“ das Anliegen des Buchs und der zugrunde liegenden Arbeiten auf recht anschauliche Weise zusammen. Im ersten dieser beiden Beiträge wird eine instruktive Perspektive auf Wissensproduktion in eher anwen- dungs- und eher forschungsbezogenen Kontexten gerichtet. Sie macht deutlich, mit welchen Dilemmata das hohe Lied der Inter- oder Transdisziplinarität in praxi konfrontiert ist. Im zweiten Beitrag wird das theoretische bzw. methodologische Setting der Forschungspraxis im Sonderforschungsbereich erläutert. Endlich erfährt man, wie genau eigentlich die praktische Arbeit in einem großen Verbundvorhaben der Einlösung der hehren Ziele auf Produktion wissenschaftlicher Exzellenz dient bzw. dienen kann. Dazu werden hier verschiedene Formen der trans- und interdisziplinären Kooperation zum Gegenstand einer wissenschaftsethnographischen Beobachtung gemacht. Auf diese Weise tritt die Blackbox der Wissensproduktion hervor, die vor allem in der großkalibrigen Verbundforschung (bezogen auf eingesetzte Ressourcen, Personal, Teilvorhaben) oft verschlossen bleibt.

Das Buch vermag ein neues, theoretisch anspruchsvolles Licht auf die vielfältigen Prozesse zu werfen, die man als Globalisierungen (Plural) spätmoderner Gesellschaften verstehen kann. Es ist nicht durchgängig frei von Setzungen aus einem essenzialistischen Diskurs, den man eigentlich zu überwinden anstrebt (etwa „Der Nationalstaat ist lange nicht mehr der primäre Raum der Politik“, S. 109; „20 Jahre nach dem Spatial Turn ...“, S. 417). Gelegentlich bedient man sich sperriger Begrifflichkeiten, wie etwa „Polykontexturalisierung“. Doch bietet das Werk insgesamt einen sehr anregenden Einblick in die vielgestaltigen Facetten seines Gegenstandes. Die Tatsache, dass es der Leserschaft als Open-Access-Publikation zur Verfügung gestellt wird, dürfte seiner weiten Verbreitung zugutekommen, womöglich auch außerhalb des akademischen Spektrums.

\section{Vollständige bibliographische Angaben des rezensierten Werkes:}

Löw, Martina; Sayman, Volkan; Schwerer, Jona; Wolf, Hannah (Hrsg.) (2021): Am Ende der Globalisierung. Über die Refiguration von Räumen. Bielefeld: Transcript Verlag. 485 Seiten.

\section{Literatur}

Rodrik, D. (2011): The globalization paradox. Why global markets, states and democracy can't coexist. Oxford.

Sheppard, E.S. (2016): Limits to globalization. Disruptive geographies of capitalist development. Oxford. 